\section{Nachhaltige Entwicklung im Energiebereich ${ }^{1}$}

\author{
Jürgen Kopfmüller, ITAS
}

\begin{abstract}
Vor dem Hintergrund der verschiedentlichen Relevanz des Energiebereichs werden in diesem Beitrag erste Schritte zur Umsetzung des Leitbilds einer global nachhaltigen Entwicklung - basierend auf dem integrativen Ansatz der HGF - für diesen Bereich skizziert. Mit Blick auf die handlungsstrategische Ebene werden dabei die Fragen hervorgehoben, die im Zusammenhang mit einer deutlich umfangreicheren Nutzung regenerativer Energieträger zu beantworten wären.
\end{abstract}

Unbestritten stellt die Verfügbarkeit von Energie bzw. von Energiedienstleistungen eine fundamentale Voraussetzung für gesellschaftliche Entwicklung, insbesondere in hochentwickelten Industriegesellschaften, dar. Zugleich sind damit jedoch erhebliche ökologische und sozioökonomische Probleme verbunden:

- $\quad$ Ein Viertel der Menschheit (ca. 1,6 Mrd. Menschen) hat keinen Zugang zu kommerziellen Energieträgern bzw. zu Strom, bis zum Jahr 2020 sollen es rund 2 Mrd. sein. In den Industriestaaten vereinen rund $20 \%$ der Menschheit für ihren relativ effizient produzierten, hohen Lebensstandard ca. zwei Drittel des Weltprimärenergieverbrauchs und $80 \%$ des Stromverbrauchs auf sich; demgegenüber verbrauchen rund $80 \%$ der Menschheit in den Entwicklungsländern für ihren ineffizienter produzierten, aber erheblich niedrigeren Lebensstandard ein Drittel der Primärenergie und $20 \%$ des Stroms. Der Pro-Kopf-Verbrauch der 2 Mrd. ärmsten Menschen liegt um den Faktor 25 niedriger als in den Industriestaaten. Vor allem in Entwicklungsländern ist der Mangel an Energie häufig mit existenziellen Problemen wie Armut oder mangelnder Grundversorgung (bzgl. Ernährung, Gesundheit, Wohnung usw.) verbunden.

- Die Bereitstellung von Nutzenergie wird 1990 bis 2020 mindestens 15.000 Mrd. US\$ für die Erstellung und Modernisierung der nach aktuellen Energieverbrauchsprog- nosen notwendigen Infrastruktur - vor alem in Entwicklungsländern und den oste uropäischen Staaten - erfordern.

- Mit der Bereitstellung und Nutzung von Energierohstoffen sowie der Entsorgung von Abfallprodukten aus Umwandlungsprozessen sind erhebliche und über teilweise sehr lange Zeiträume wirksame Gefährdungen der natürlichen Lebensgrundlagen (durch Schadstoffemissionen, Klimaveränderungen, Ressourcenverbrauch usw.) und der menschlichen Gesundheit sowie Risiken in Form von Unfällen verbunden.

All dies gilt in besonderem Maße angesichts der globalen Prognosen zum fortdauernden Bevölkerungs- und Wirtschaftswachstum. Daraus wird unmittelbar deutlich, dass die globale Realisierung des Nachhaltigkeitsleitbilds in hohem Maße von der Entwicklung im Energiesektor abhängt bzw. dass der Umsetzung des Leitbilds in diesem Sektor entscheidende Bedeutung zukommt.

\section{Allgemeine Handlungsleitlinien}

Ausgangspunkt für die nachfolgend skizzierte Übertragung des Nachhaltigkeitsleitbilds auf den Energiebereich ist der integrative Ansatz, der im Rahmen des HGF-Verbundprojekts „Global zukunftsfähige Entwicklung - Perspektiven für Deutschland“" entwickelt wurde ${ }^{2}$. Hierin erfolgt der Einstieg in die Operationalisierung des Leitbilds nicht - wie in den bislang vorliegenden Ansätzen - über die einzelnen Dimensionen bzw. „Säulen“ (Ökonomie, Ökologie, Soziales), sondern über als konstitutiv eingestufte Elemente nachhaltiger Entwicklung wie sie auch dem Brundtland-Bericht, den RioDokumenten sowie den Dokumenten des RioFolgeprozesses zugrunde liegen: das Postulat der gleichberechtigten inter- und intragenerativen Gerechtigkeit, die globale Orientierung sowie die anthropozentrische Perspektive. Diese werden in drei generelle Ziele nachhaltiger Entwicklung ,übersetzt“ - die Sicherung der menschlichen Existenz, die Erhaltung des gsellschaftlichen Produktivpotenzials und die Bewahrung der Entwicklungs- und Handlungsmöglichkeiten einer Gesellschaft - die wiederum durch eine Reihe von Nachhaltigkeitsregeln bzw. -leitlinien konkretisiert wer- 
den, die die wesentlichen ökologischen, sozialen, ökonomischen und institutionellen Aspekte nachhaltiger Entwicklung reflektieren.

Ausgangsthese ist dabei, dass ein die globalen Aspekte angemessen reflektierendes Konzept die beiden Komponenten Bestandssicherung einerseits und Entwicklung andererseits (letztere verstanden als Verbesserung von Lebensbedingungen wie auch als Veränderung oder Anpassungsfähigkeit) gleichberechtigt berücksichtigen muss. Ob und wie sich diese beiden Zielsetzungen grundsätzlich und unter Energieaspekten in Einklang bringen lassen, ist umstritten. Von zahlreichen Experten wird eine Bestandserhaltungsstrategie der Industriestaaten bei gleichzeitigem Aufholen der Entwicklungsländer unter Nachhaltigkeitsaspekten als langfristig nicht möglich bzw. nicht verantwortbar eingeschätzt. Daher wird die Frage zu stellen sein, welche Konsequenzen sich daraus für das Entwicklungsmodell der Industriestaaten ergeben können bzw. müssen.

Die Relevanz des Energiebereichs betrifft hier insbesondere die Frage, inwieweit für eine solche globale Entwicklungsstrategie Energieressourcen wie lange ausreichend vorhanden wären und welche Folgen ihre Nutzung hätte. Im Zuge des erforderlichen gesellschaftlichen Diskussionsprozesses über das Nachhaltigkeitsleitbild ist es möglich und erforderlich, ein zumindest aus heutiger Sicht als angemessen bewertetes Anforderungsprofil für Nachhaltigkeit im Energiesektor zu erarbeiten, das es erlaubt, im Sinne des intra- und intergenerativen Gerechtigkeitspostulats global hinreichende Handlungsspielräume für die Menschheit zu gewährleisten.

Auf der inter- bzw. supranationalen Ebene sind derartige Leitlinien schon vereinzelt formuliert worden (siehe insbesondere UNECOSOC 2000; UNDP/SEI/UNCSD 1997; UNDP/Un-ECOSOC/WEC 2000). In Deutschland hat $u$. a. das ,Forum für Zukunftsenergien“ $\mathrm{zu}$ dieser Frage Stellung genommen (vgl. M. Eichelbrönner, H. Henssen 1998). Darauf und auf der bisherigen Konkretisierung des integrativen HGF-Ansatzes aufbauend werden nachfolgend allgemeine Handlungsleitlinien benannt:

Ressourcenschonung: Der Verbrauch energetischer und nicht-energetischer Ressourcen bei der Förderung, Umwandlung und Nutzung von Primärenergieträgern ist zu minimieren.
Umwelt-, Klima- und Gesundheitsverträglichkeit: Überlastungen der Regenerations- und Anpassungsfähigkeiten der Ökosysteme sowie Gefahren für die menschliche Gesundheit sind zu vermeiden.

Soziale Gerechtigkeit: Allen Menschen sind vergleichbare Chancen des Zugangs zu Energieressourcen bzw. -dienstleistungen zu gewährleisten. Bei der Gestaltung von Energieversorgungssystemen ist allen Betroffenen die Teilhabe an den Entscheidungsprozessen zu ermöglichen.

Versorgungssicherheit: Die zur Bedürfnisbefriedigung der Menschen erforderlichen Energiedienstleistungen und die dafür notwendigen Versorgungsinfrastrukturen müssen dauerhaft zur Verfügung stehen.

Risikoarmut und Fehlertoleranz: Risiken bei Förderung, Nutzung und Entsorgung sind $\mathrm{zu}$ minimieren und in ihrer räumlichen und zeitlichen Ausdehnung zu begrenzen. Dabei sind auch fehlerhaftes Verhalten, unsachgemäße Handhabung oder mutwillige Zerstörung zu berücksichtigen.

Wirtschaftliche und soziale Verträglichkeit: Das Energiesystem ist zu vertretbaren einzel- und gesamtwirtschaftlichen Kosten sowie sozial verträglich zu gestalten.

Internalisierung externer Kosten: In allen Phasen energiebezogener Aktivitäten (Förderung, Umwandlung usw.) sind die externen ökologischen und sozialen Kosten zu internalisieren.

Internationale Kooperation: Die Gestaltung der globalen Energiesysteme muss auf der Basis internationaler Kooperation erfolgen.

Gemäß der konzeptionellen Logik des HGF-Ansatzes sind diese Leitlinien als Gesamtheit zu betrachten, d. h. jede gilt im Prinzip nur in den Grenzen aller anderen. Sie sind als Mindestanforderungen zu verstehen, was bedeutet, dass andere, durchaus wünschbare Elemente wie Wachstum oder Luxuskonsum zwar nicht grundsätzlich ausgeschlossen, jedoch insoweit als nachrangige bzw. abhängige Größen zu betrachten sind, als sie nur in dem Maße zulässig sind, wie sie die Einhaltung der Mindestbedingungen nicht gefährden. 


\section{Formulierung von Handlungszielen}

Es sind vor allem zwei Argumente, die bei der Umsetzung dieser Handlungsleitlinien für die Setzung konkreter quantitativer oder qualitativer Ziel- oder Richtungsvorgaben für bestimmte, die Leitlinien konkretisierende Leitindikatoren sprechen. Sie dienen erstens der stets geforderten Erhöhung der Planungssicherheit der wirtschaftlichen Akteure. Zweitens liefern nur konkrete Ziele Referenzwerte, die es ermöglichen, bestehende Ist/Soll-Diskrepanzen hinsichtlich nachhaltiger Entwicklung zu identifizieren, um daraus Zukunftsszenarien sowie geeignete Handlungsstrategien ableiten und bewerten zu können. Ergänzend bzw. alternativ zur Setzung fester Zielwerte wird auch über sogenannte „Leitplanken“ diskutiert, die für bestimmte Indikatoren quasi obere und untere „Grenzen“ eines jeweils „,tragfähigen Möglichkeitsraums" definieren sollen.

Bislang sind solche Ziele vorwiegend zur Emissions- und deutlich weniger zur Ressourcenproblematik realisiert oder in der Diskussion. Sie sind Gegenstand, zum Teil auch Ergebnis nationaler und internationaler Aushandlungsprozesse unter Beteiligung wichtiger gesellschaftlicher Gruppen. Als Kriterien für die Zielfestlegung kommen zum einen das Vorsorge-, Verursacher- und Kooperationsprinzip zum tragen, zum anderen Aspekte der wirtschaftlichen, sozialen und technologischen Praktikabilität (etwa im Hinblick auf die Verteilung finanzieller Lasten zwischen Staaten oder Regionen).

Emissionsbezogene Ziele existieren zum einen im Bereich der Luftschadstoffemissionen, wo das unter der Federführung der UN Economic Commission for Europe (UN-ECE) 1999 entstandene „Göteborg-Protokoll zur Vermeidung von Versauerung, Eutrophierung und bodennahem Ozon" die aktuellen Reduktionsvorgaben für einzelne Staaten bis zum Jahr 2010 für bestimmte Schadstoffe enthält. Den zweiten zentralen Bereich stellen die Treibhausgasemissionen dar, für die auf der Basis der Klimarahmenkonvention von 1992 das Kyoto-Protokoll von 1997 und seine Konkretisierung den Rahmen für Staaten oder Staatengruppen bildet. Über dessen Zeithorizont hinaus hat insbesondere die Enquête-Kommission des 12. Deutschen Bundestags „Schutz der Erdatmosphäre" bezogen auf die Industriestaaten Energie- und $\mathrm{CO}_{2}$-bezogene Reduktionsziele formuliert.

Die selteneren, energieressourcenbezogenen Ziele sind vorwiegend auf den Anteil regenerativer Energieträger (REG) am Cesamtmix und auf die Energieeffizienz (definiert als Energieverbrauch pro BIP) beschränkt. Solche REG-Quoten sagen jedoch nichts über die absolute Höhe des Verbrauchs nichterneuerbarer Energieträger aus, das Effizienzkriterium wenig über den Gesamtenergieverbrauch, der wesentlich vom Umfang der insgesamt erzeugten Wirtschaftsleistung abhängt (Stichwort ,,rebound-Effekt", d. h. Kompensation von Effizienzgewinnen durch $\mathrm{Zu}$ wachs bei Produkten und deren Nutzung). Deswegen halten wird in Ergänzung Ziele für erforderlich, die sich auf den Umfang des absoluten Ressourcenverbrauchs oder, besser, auf die Reichweite von (Energie)Ressourcen (definiert als verfügbare Ressourcenmenge dividiert durch den Verbrauch pro Jahr, gemessen in Jahren) beziehen. Trotz vielfach diskutierter Einschränkungen des Reichweiten-Indikators, z. B. aufgrund schwieriger Abgrenzungen zwischen „Ressourcen“ und „Reserven“ sowie begrenzter Datenverfügbarkeit, könnte damit dem im Nachhaltigkeitsleitbild zentralen Verantwortungsprinzip für kommende Generationen am ehesten Rechnung getragen werden.

In der nachfolgenden Tabelle 1 sind für die zwei „klassischen“ Handlungsleitlinien Indikatoren und Ziele angeführt. 
Tab. 1: Leitlinien, Indikatoren und Ziele

\begin{tabular}{|c|c|c|}
\hline Leitlinie & Leitindikator & Ziel \\
\hline $\begin{array}{l}\text { Ressourcen } \\
\text { schonung }\end{array}$ & 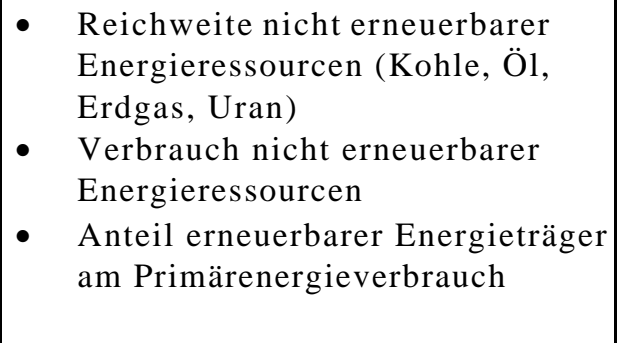 & $\begin{array}{l}\text { Darf nicht sinken (bzw. nur um best. } \\
\text { Prozentsatz in best. Zeitraum); } \\
\text { Minus } 20 \% \text { bis } 2010 \text { (Schweiz); } \\
\text { Verdoppelung der Verbrauchseffi- } \\
\text { zienz bis } 2020 \text { (Enquete- } \\
\text { Kommission); } \\
\text { Verdopp. Bis } 2010 \text { (Bundesreg.); } \\
\text { Anteil von } 50 \% \text { bis } 2050 \text { (BMU) }\end{array}$ \\
\hline $\begin{array}{l}\text { Umwelt-, Klima- } \\
\text { und Gesundheits - } \\
\text { verträglichkeit }\end{array}$ & $\begin{array}{l}\text { - } \mathrm{CO}_{2} \text {-Emissionen (alternativ } \mathrm{CO}_{2} \text { - } \\
\text { Äquivalente oder „Kyoto-Korb“) } \\
\text { - } \mathrm{SO}_{2} \text { - bzw. } \mathrm{NO}_{\mathrm{x}}-\text { Emissionen }\end{array}$ & $\begin{array}{l}\text { Deutschland: Anteil von } 21 \% \text { an den } \\
8 \% \text { Reduzierung bezogen auf EU } \\
\text { (Kyoto-Protokoll); } \\
\text { minus } 25 \% \mathrm{CO}_{2} \text { bis } 2005 \text { (zu 1990) } \\
\text { (Bundesregierung) } \\
\text { minus } 90 \% \text { bzw. minus } 60 \% \text { bis } \\
2010 \text { („Göteborg-Protokoll“) }\end{array}$ \\
\hline
\end{tabular}

Solche Indikatoren und Ziele sind in der Logik des hier skizzierten Konzepts auch für die Leitlinien notwendig, für die bislang kaum Vergleichbares existiert. Beispielsweise wäre hinsichtlich des Aspekts der sozialen Gerechtigkeit der Anteil der Menschen ohne Zugang zu Energie(dienstleistungen) bedeutsam. Eine denkbare Zielgröße könnte hier - in Anlehnung an die von verschiedenen UN-Organisationen gesetzten Entwicklungsziele zu Problemfeldern wie Kindersterblichkeit, Unterernährung oder Analphabetismus - die Halbierung dieses Anteils bis zum Jahr 2020 sein. Zum anderen müssten Ziele für Form und Umfang der Einbeziehung relevanter Gruppen in Entscheidungsprozesse zur Gestaltung von Energieversorgungssystemen konkretisiert werden. Leitindikatoren zur wirtschaftlichen und sozialen Verträglichkeit könnten der Anteil der Energiekosten im produzierenden Gewerbe sein (evtl. Ziel: im Durchschnitt nicht über $5 \%$ ), der Anteil der Ausgaben für Energie (Strom, Heizung, Mobilität) in privaten Haushalten mit geringem Einkommen (evtl. Ziel: nicht über $15 \%$ ) oder die Diversifizierung bei den Energieimport-Lieferländern (Ziel: Der Anteil der größten drei Länder darf z. B. 30 oder $50 \%$ nicht übersteigen).

Bezogen auf die Internalisierungsleitlinie müssten sich idealtypische Indikatoren und Ziele auf den Umfang der Einbeziehung der verschiedenen externen Kosten vor allem in die Preisgestaltung für Energie(dienstleistungen) beziehen. Da dies datenbedingt nur schwer möglich sein dürfte, wäre quasi als indirekter Indikator etwa der Aufkommensanteil energiebzw. ressourcen- oder emissionsbezogener Steuern am gesamten Steueraufkommen denkbar. Schließlich wären für die Kooperationsleitlinie Ziele zu erwägen, die sich etwa auf den Anteil der Ausgaben für nachhaltigkeitsfördernde, energiebezogene Projekte oder Forschungsaktivitäten beziehen (gemessen am Gesamtetat für Entwicklungszusammenarbeit bzw. am energiebezogenen Entwicklungsetat) oder auf Art, Umfang und regionale Differenzierung energiebezogener Technologietransferaktivitäten.

\section{Die handlungsstrategische Ebene}

Um diese Ziele erreichen zu können, sind eine Fülle von Strategien, Maßnahmen und Instrumenten denkbar, die in unterschiedlicher Weise schon praktiziert oder meist noch diskutiert werden. Greift man das bislang am stärksten bearbeitete Feld des Ressourcen-, Umwelt- und Gesundheitsschutzes heraus, existieren grundsätzlich drei Strategieansätze:

Effizienz: Im Vordergrund steht hier die rationell(er)e Energiewandlung bzw. -verwendung sowie die Substitution zwischen Energieträgern mit unterschiedlichem Energiegehalt ${ }^{3}$. Ziel ist die Verringerung des energetischen und stofflichen Aufwands zur Erstellung der bestehenden 
(Energie)Dienstleistungen (z. B. einer Raumtemperatur von $21^{\circ} \mathrm{C}$ ).

Konsistenz: Ziel ist hier die Substitution des mit Nachhaltigkeitszielen unverträglichen Verbrauchs fossiler Energieträger durch regenerierbare Energieträger (REG) sowie die Vermeidung der Nutzung risikoreicher Energiesysteme zur Erstellung bestehender Dienstleistungen.

Suffizienz: Zielsetzung ist hier die Reduzierung bzw. Veränderung der dem Energieverbrauch zugrunde liegenden menschlichen Aktivitäten, Dienstleistungen und Bedürfnisse, also die Änderung von Lebensstilen (z. B. die Reduzierung der Raumtemperatur unter $21^{\circ} \mathrm{C}$ ).

Denkbare handlungsstrategische Alternativen seien an einem Beispiel verdeutlicht: Die heutigen durchschnittlichen Pro-Kopf-Energieverbräuche liegen bei ca. 7,5 t SKE ${ }^{4}$ pro Jahr in den Industriestaaten und ca. 0,9 t SKE in den Entwicklungsländern. Prognostiziert wird vom WEC $^{5}$ für das Jahr 2050 - unter bestimmten Annahmen zu Bevölkerung (Anstieg von derzeit 6 auf 9 Mrd., davon $85 \%$ in Entwicklungsländern lebend), Wirtschaftswachstum (ca. $2 \%$ pro Jahr) und Energieeffizienz (Steigerung um 0,5 bis $1 \%$ pro Jahr) - ein geringer Rückgang in den Industriestaaten auf ca. 7,2 t SKE und eine Steigerung auf 2,2 t SKE in den Entwicklungsländern, was insgesamt zu einer ungefähren Verdoppelung des globalen Primärenergieverbrauchs führen würde.

Geht man stattdessen von der - vielfach geteilten - langfristigen Zielorientierung einer tendenziellen Angleichung der globalen ProKopf-Energieverbräuche auf erheblich reduziertem Gesamtverbrauchsniveau aus, würde dies implizieren, dass die Industriestaaten ihren Pro-Kopf-Verbrauch reduzieren müssten (da eine globale Angleichung auf deren hohem Niveau dauerhaft nicht tragfähig wäre) und dass zugleich den Entwicklungsländern $\mathrm{Zu}-$ wächse eingeräumt werden. Ein mögliches Szenario könnte dann wie folgt aussehen: Einer Halbierung der Pro-Kopf-Verbräuche in den Industriestaaten auf ca. 3,5 t SKE würde eine Steigerung in den Entwicklungsländern auf 2,2 t SKE gegenüberstehen. Die daraus resultierende Zunahme des globalen Energieverbrauchs um ungefähr $60 \%$ wäre mit dem anvisierten Ziel einer Reduzierung der globalen $\mathrm{CO}_{2}$-Emissionen um $50 \%$ dann in Einklang zu bringen, wenn regenerative Energien einen Anteil von rund $60 \%$ ausmachen würden. Würde ein so hoher Anteil regenerativer Energien nicht gewünscht (weil etwa mit zu hohen Kosten oder mit unerwünschten Nebenfolgen in Verbindung gebracht), wären im Prinzip als Alternativen bzw. Ergänzungen eine noch weiter gesteigerte Energieeffizienz und/oder ein geringeres Wirtschaftswachstum und/oder ein geringerer Bevölkerungszuwachs denkbar ${ }^{6}$.

Vor diesem Hintergrund halten wir zur Erreichung der schon existierenden und der darüber hinaus diskutierten Ziele - unter Ausklammerung der höchst brisanten Bevölkerungsthematik - ein gestuftes Vorgehen für sinnvoll: Erste Priorität besitzt die Realisierung der vorhandenen bzw. erkennbaren Effizienzund Konsistenzsteigerungspotenziale, soweit dies zu vertretbaren „Kosten“ möglich ist. In Studien etwa von Shell oder vom WEC wird davon ausgegangen, dass sowohl die angedeutete Größenordnung eines Einsatzes regenerativer Energien als auch Energieeffizienzsteigerungen von 2 - $3 \%$ pro Jahr im Prinzip - zlmindest aus technischer bzw. potenzialseitiger Sicht - bis 2050 realisierbar wären. Insoweit wie diese Strategien nicht ausreichen, müssten weitergehende suffizienzorientierte Ansätze und entsprechend ausgerichtete Instrumente zur Anwendung kommen.

In der guten Tradition systemanalytischer, ganzheitlicher Betrachtungen sind dabei im Hinblick auf eine derart breite Nutzung regenerativer Energien einige Fragen vertieft $\mathrm{zu}$ untersuchen bzw. hierzu schon vorliegende Antworten zu bewerten:

- Wie sind die Potenziale der Nutzung regenerativer Energien im Detail beschaffen?

- global gesehen, in Deutschland;

- technisch, ökonomisch;

- differenziert nach einzelnen Energieträgern oder nach Aktivitätsfeldern (z. B. Mobilität, Wohnen/Bauen usw.) als mögliche Einsatzbereiche für $\mathbf{e}$ generative Energien.

- Welche Konflikte (z. B. hinsichtlich Flächennutzung) können entstehen und nach welchen Kriterien sind sie zu behandeln?

- Welche Beiträge können regenerative Energien zur Erreichung verschiedener Nachhaltigkeitsziele im Energiebereich leisten? 
- Welche Erfordernisse und Folgen sind mit der Realisierung dieser Potenziale verbunden (z. B. hinsichtlich Infrastrukturen, Kosten, Umwelt- und Flächenverbräuche usw.)?

- Mit welchen Instrumenten kann die Nutzung der als ,nachhaltig“ einzustufenden Potenziale am besten gefördert werden (Erneuerbare-Energien-Gesetz (EEG), Quoten- oder Ausschreibungsmodelle, Energie-/ $/ \mathrm{CO}_{2}$-Steuer, ordnungsrechtliche Instrumente verschiedener Art)?

- Welche Rolle spielen generelle Rahmenbedingungen wie z. B. Globalisierungsprozesse oder Liberalisierungen (d. h. die Deregulierung und marktliche Organisation insbesondere der leitungsgebundenen Energien und die damit verbundene Öffnung für den internationalen Wettbewerb)? Was bewirken sie und wie ist damit umzugehen?

$\mathrm{Zu}$ einigen dieser Fragen werden die nachfolgenden Beiträge in unterschiedlicher Ausführlichkeit Stellung beziehen.

\section{Anmerkungen}

1) Die nachfolgenden Ausführungen beruhen im Wesentlichen auf einer umfangreichen schriftlichen Stellungnahme, die anlässlich der Anhörung der Enquete-Kommission des 14. Deutschen Bundestages "Nachhaltige Energieversorgung unter den Bedingungen der Globalisierung und der Liberalisierung " am 19.9.2000 ausgearbeitet wurde; vgl. J. Kopfmüller, R. Coenen, J. Jörissen (unter Mitarbeit von T. Fleischer, C. Rösch, G. Sardemann, V. Schulz) (alle FZK-ITAS); O. Langniß, J. Nitsch (beide DLR, TT-STB): „Konkretisierung und Operationalisierung des Leitbilds der nachhaltigen Entwicklung für den Energiebereich“, FZKA Nr. 6578, Karlsruhe 2000.

2) Vgl. J. Jörissen et al.: Ein integratives Konzept nachhaltiger Entwicklung: Der theoretischkonzeptionelle Ansatz des HGF-Verbundprojektes. In: TA-Datenbank-Nachrichten, Nr. 2/2000, S. 35-42; J. Kopfmüller et al.: Nachhaltige Entwicklung integrativ betrachtet. Konstitutive Elemente, Regeln, Indikatoren; edition Sigma, Berlin 2001 (in Druck).

3) Daneben wäre hier auch die energetische Nutzung von solchen Rest- und Abfallstoffen zu nennen, die nicht vermeidbar sind oder die nicht wiederverwertet bzw. in den stofflichen Kreislauf zurückgeführt werden können.

4) $\mathrm{SKE}=$ Steinkohleeinheiten

5) World Energy Council/Welt-Energie-Rat

6) Unter der Annahme, dass ein in diesen GröBenordnungen erforderlicher Ausbau der Kernenergie gesellschaftlich nicht akzeptanzfähig wäre.

\section{Literatur}

Eichelbrönner, M.; Henssen, H., 1998: Langfristige Aspekte der Energieversorgung. Ergebnisse eines Diskussionsprozesses. In: Energiewirtschaftliche Tagesfragen, Vol. 48, Nr. 8, S. 496-500

UNDP (United Nations Development Programme); SEI (Stockholm Environment Institute); UNCSD (United Nations Commission on Sustainable Development), 1997: Energy after Rio: Prospects and Challenges. New York

UNDP; UNDESA (United Nations Department of Economic and Social Affairs); WEC (World Energy Council), 2000: Energy and the Challenge of Sustainability. New York

UN-ECOSOC (United Nations-Economic and Social Council), 2000: Energy and Sustainable Development: Key Issues. Preparation for the ninth Session of the Commission on Sustainable Development by the Intergovernmental Group of Experts on Energy and Sustainable Development; Document No. E/CN.17/ESD/2000/3, New York

\section{Kontakt}

Dipl.-Volksw. Jürgen Kopfmüller Forschungszentrum Karlsruhe $\mathrm{GmbH}$

Institut für Technikfolgenabschätzung und Systemanalyse (ITAS)

Postfach 3640, 76021 Karlsruhe

Tel.: +49 (0) 7247 / 82 - 4570

Fax: +49 (0) 7247 / 82 - 4806

E-Mail: Kopfmueller@itas.fzk.de

URL: www.itas.fzk.de 